\title{
The Semantic Structures of Chinese Verb-resultatives
}

\author{
Hongling Yin \\ School of Foreign Languages \& Cultures, Nanjing Normal University \\ 122 Ninghai Road, Nanjing 210097, China \\ E-mail: honglin1975@gmail.com
}

Received: April 17, 2011 Accepted: May 8, $2011 \quad$ doi:10.5539/ijel.v1n2p126

This is the project financed by the Priority Academic Program Development of Jiangsu Higher Education Institutions, and Jiangsu Education Department's Higher School Philosophy and Social Science Research Foundation (No. 2011SJB740011)

\begin{abstract}
The theory of verb-assignment in the traditional argument structure cannot offer a satisfactory explanation for the specific grammatical structures and semantic features of Chinese verb-resultative constructions. By distinguishing the conceptual content from the grammatical meaning and representing the sentence-level conceptual content in Conceptual Frames, the grammatical realization rules of verb-resultative constructions can be analyzed more accurately. The conceptual frame of a verb-resultative construction is mainly composed of action conceptual structure and causation conceptual structure; and the participants in these two conceptual structures will be conflated and realized as the relevant argument roles in the semantic structure. When the Causee is conflated with different participants in the action conceptual structure, it can be construed in different ways, and realized in different positions in the corresponding semantic and grammatical structures, thereby ultimately influencing the whole configuration of the verb-resultative construction.
\end{abstract}

Keywords: Chinese verb-resultative Constructions, The semantic structure, The conceptual frame, Causee

\section{Introduction}

As a language structure in which the resultative phrase describes the state change of an object caused by the action denoted by the main verb (cf. Levin, 1993; Goldberg, 1995; Boas 2003), the verb-resultative construction, enjoys an independent grammatical status. It is formally presented as " $\mathrm{N} 1+\mathrm{V}+\mathrm{RP}(+\mathrm{N} 2)$ ") $(\mathrm{RP}=$ resultative phrase, $\mathrm{N} 2$ is optional). The following sentences are the typical Chinese resultatives:

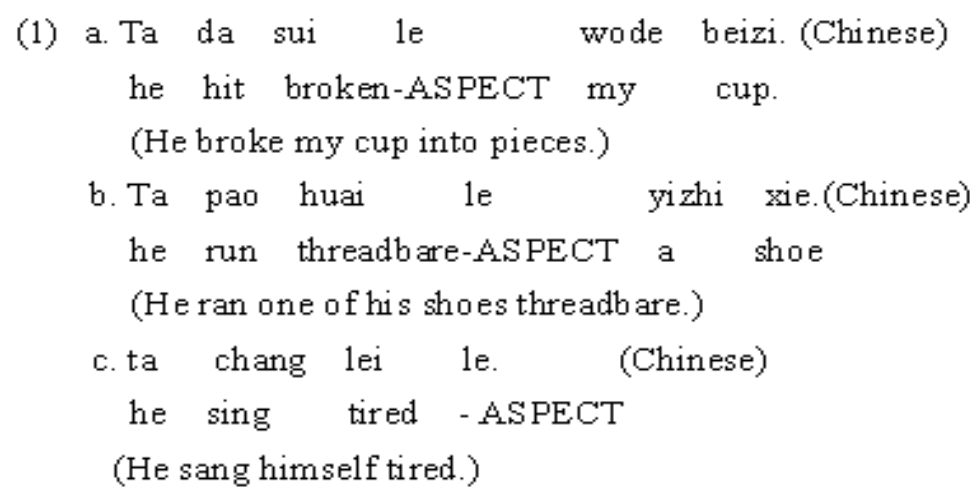

Goldberg (1995: 10) points out the particular semantic (or argument) structures of verb-resultative constructions pose a great challenge to the traditional verb-assignment theory (cf. Fillmore, 1971; Chomsky, 1981). Meanwhile, the Lexical Semantics (Pinker, 1989; Levin, 1993; Boas, 2003) also adopts the verb-centered methodology involving all usages into the lexical-feature description of the corresponding verb - in dealing with the clause-level semantic structures, which makes the lexicon over-loaded and unworkable. In fact, as a gestalt construction which enjoys independent grammatical status, the verb-resultative construction has its own special semantic structure and argument-realization rules. This paper aims to prove that the theory of Conceptual Frames can be applied to offer a clearer and more accurate analysis to the features and realization rules of Chinese 
verb-resultatives.

\section{Challenges Posed by Chinese Verb-resultatives}

From the examples above, we can see that not all the verb-resultative constructions share the exactly same syntactic or semantic structures. Both (1a) and (1b) have two (noun) arguments (two-place), while (1c) has only one argument(one-place). Moreover, in the two-place verb-resultative constructions, the noun phrase following the resultative phrase can be the selected object of the main transitive verb (as in 1a), the unselected object of the transitive verb(as in (2)), or the unselected object of the intransitive verb(as in (1b)).

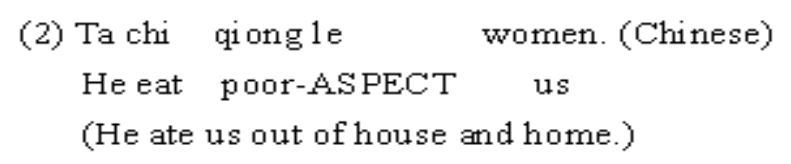

The semantic structures pose lots of challenges to the traditional theory of argument structure. First, if it is the main verb that determines the number of arguments and assign thematic roles, then the direct object in (2) should be the thing being eaten, but "women" (us) has no logical relation with the verb "chi" (eat). Besides, the verb "pao" (run) is an intransitive verb, but it is followed by "yizhi xie"(a shoe)(1b). How can the noun phrase be allowed and what thematic role is it assigned? Lexicalists propose that the noun phrases in the syntactical object position in such sentences as $(1 \mathrm{~b}, 2)$ can be allowed by involving them into the feature-description of the relative verb. But we can see that the referents of objects (underlined) in sentences (3) play different semantic roles in the related verbal event structures. To allow them, a set of restriction rules must be set, which is bound to make the theoretical system too complicated to be workable.
(3) a. He swept the room clean.
(Actee)
b. He swept the crumbs off the floor.
(Entity removed)
c. He swept the broom to pieces.
(Instrument)
d. He swept himself to exhaustion.
(Actor as a whole)

The second problem is whether a noun phrase is only assigned a thematic role? That is, is the $\theta$-criterion strictly observed? The answer is "yes" in GB theory and LFG (such as the principle of biuniqueness). But this is challenged by Jackendoff (1990: 59-61) and others: an NP can have more than one $\theta$-role, and multiple NPs may hold a single $\theta$-role. This is more obvious in verb-resultative constructions. Please consider the following sentence:

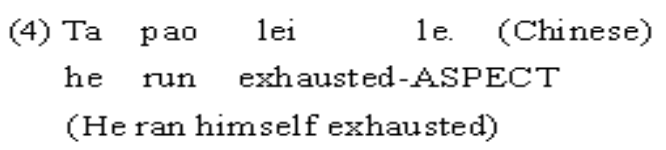

What thematic role is assigned to "ta"(he), one or two? According to the principle of $\theta$-role assignment, every NP in a clause is assigned one and only one thematic role, but here "ta" is the agent of "pao", and at the same time the experiencer of "lei".

The last question here is the fake reflective object. The reflective pronouns, co-indexed with the subjects in (5a, $5 b)$ are not the selected objects of the main verbs. Why aren't they omitted and the verb-resultatives realized as one-place semantic structures? On the contrary, why can't the reflective pronouns(in 5c), also co-indexed with subjects, be used as the objects?

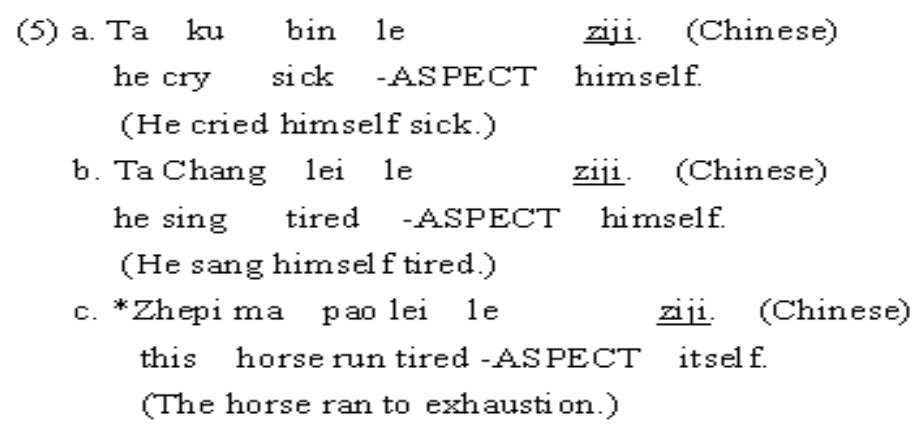

The questions above can not be given a satisfactory answer in the frame of traditional argument structure. 


\section{The Semantic Structures of Chinese Verb-resultatives}

The verb-resultatives, as a group of gestalt constructions, have their own particular semantic structures. Goldberg (1995:189) represents the typical argument structure of English verb-resultatives as <agent patient result-goal>. In this way, the typical Chinese verb-resultatives can be represented as <agent result-goal patient $>$, and they have other types of argument structures.

\subsection{The Conceptual Content and the Grammatical Meaning}

The linguistic system is language-symbol relation system, a neural cognitive system connected by the realizational relation between the conceptual meaning and the language expression (Lamb 2004). The conceptual meaning consists of the conceptual content and the grammatical meaning, and the language expression refers to the lexico-grammatical structure or phonology. The conceptual meaning is encyclopedia, connected with humans' sensory-motor system, while the sentence-level grammatical meaning includes the semantic structure and its arguments. The multi-dimensional conceptual content, via the one-dimensional grammatical meaning, is realized as the corresponding lexico-grammatical structures or phonology; that is, the grammatical meaning, which is directly related to the grammatical structure, is a bridge between the conceptual meaning and the language expression. Certainly, the three layers are bi-directionally activated. For instance, the conceptual content can activate certain grammatical meaning; meanwhile, the grammatical meaning can also activate the richer conceptual meaning. Our humans' faculty of construal plays a big role in the process of realizing the conceptual content into the grammatical meaning. The so-called construal refers to the humans' cognitive ability of conceptualizing the same scene in different ways. Langacker (1998:4) proposes that there are two variants to determine meaning: the conceptual content and the way the conceptual content is construed. Consider the next two sentences

\section{(6) a. The bottle is half empty. \\ b. The bottle is half full.}

The two sentences express the exactly same conceptual content, but the different construal results come from different locational perspectives: (6a) shows the up-to-down view; and (6b) the down-to-up, or from the different mental perspectives or expectations, which are realized as different lexical concepts: "empty" and "full". As a part of the conceptual meaning, the semantic structure is not only directly related to the grammatical structure, but also represents humans' construal result of the basic conceptual content in a language. The grammatical meaning of a clause can be formally represented by the semantic structure, while the conceptual content by the conceptual frame (cf. Cheng 2006). Take the sentence "Fred wiped the table clean" (see Table 1) From the Conceptual Process, we can see that the conceptual content activated by the sentence above contains the conceptual structure of action (Actor ${ }_{\text {Fred }}+$ Action $_{\text {wash }}+$ Actee $_{\text {the table }}$ ) and the conceptual structure of causation

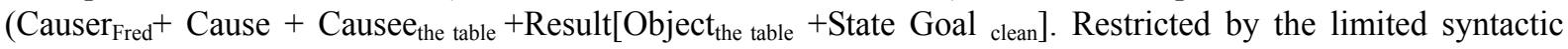
space, some participants in these two conceptual structures must be conflated and realized as some arguments in the semantic structure. In this case, the actor is conflated with the causer, realized as the agent in the semantic structure, and subject noun ("Fred") in the grammatical structure; the actee is conflated with the cause, realized as the patient in the semantic structure, and object noun phrase ("the table") in the grammatical structure. The action is conflated with the causation, realized as the predicate in the grammatical structure, and the result is realized as the Result-Goal argument, realized as the adjective phrase following the object noun phrase.

\subsection{Causation and Non-Causation}

The division of Causation and non-causation is not based on the objective condition. Despite the fact that everything the physical world is in the cause-and-effect chain, where any change has its cause, in the humans' languages, the same objective thing can be construed in different ways, as in (7):

(7) a. The vase broke.

(non-causation)

b. She broke the vase. (causation)

Although the fact that "the vase was broken" has its causer, it is not profiled and the event is construed as non-causation in (7a). But in (7b), it is "she" who causes "the vase" to undergo a change of state; the causer is profiled and the event is construed as causation. In the grammatical meaning of verb-resultatives, there exist four types of construal results: the pure auto-change(as in $8 \mathrm{a}$ ), the auto-change through action( $8 \mathrm{~b}$ ), the pure causation(8c), and the caused-change through action(8d). 


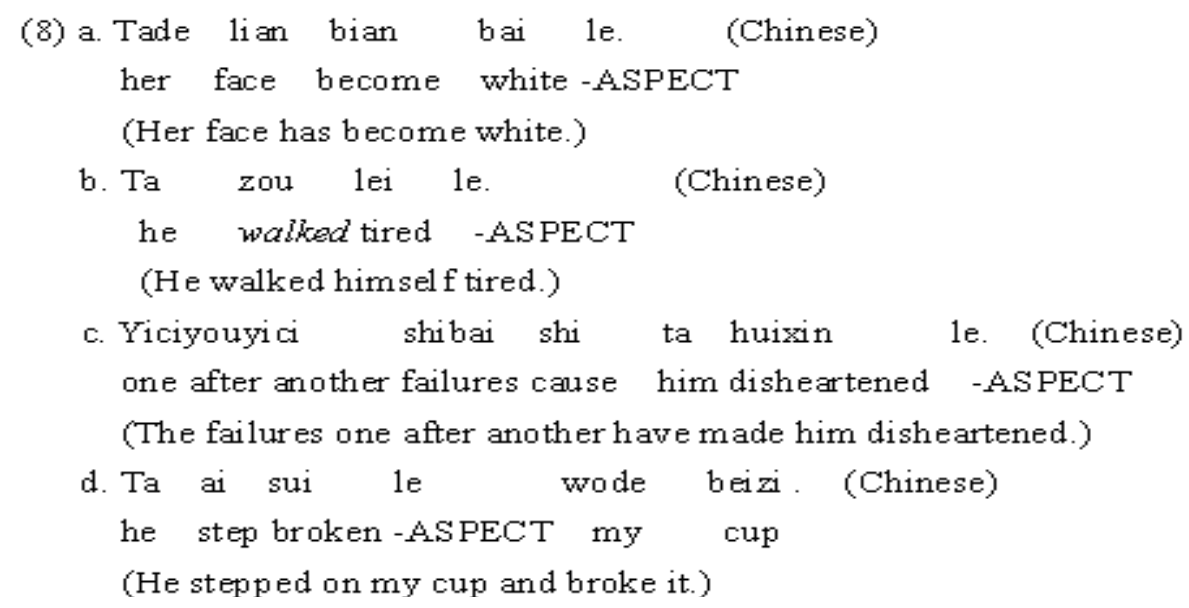

The so-called pure auto-change refers to the situation in which the conceptual structure of action is not realized in the sentence, and the predicates are some "change" verbs, such as "bian" (become). In the auto-change through action, the conceptual structure of action is realized, and the state change of the object is caused by the action. The semantic structure of the pure causation only profiles the conceptual structure of causation, and the predicates are some causation verbs like make, let, drive, render. The most typical verb-resultatives are the caused-change through action, in which the conceptual structure of action and the conceptual structure of causation are both realized in the semantic structure. The pure auto-change and the auto-change through action are realized as one-place verb-resultatives(as in $8 \mathrm{a}, 8 \mathrm{~b}$ ), and the pure causation is two-place(as in $8 \mathrm{c}$ ). The caused-change through action can be realized in various semantic structure configurations, which are greatly influenced by the selection and realization of the role of Causee.

\subsection{The Selection of Causee}

An action process often involves a variety of participants, among whom the most influential one is the Actor, who will cause other participants and even itself to undergo a change of state. Take the verb event of "he"(drink) as an example:

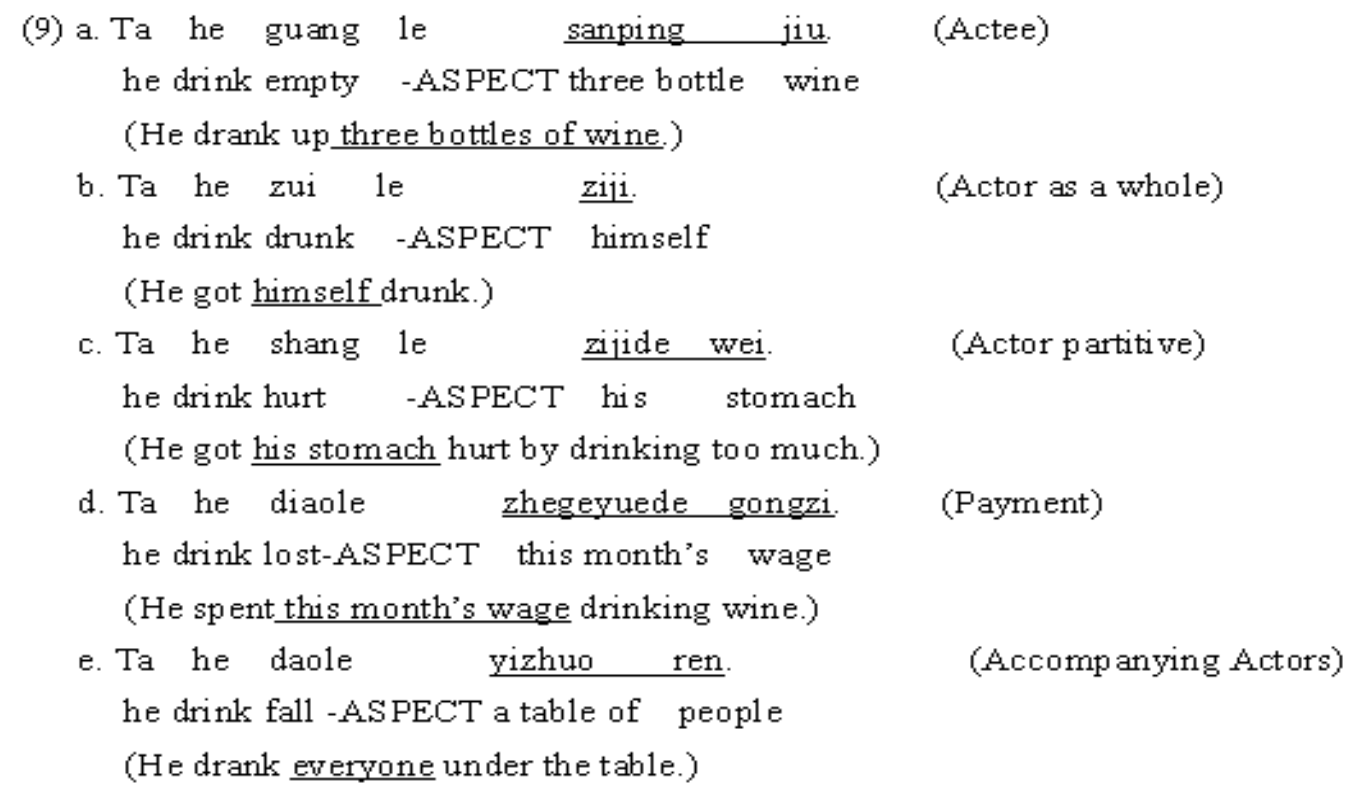

The Causee in the caused-change through action can also be conflated with the Entity Goal(the entity wanted, including the one created by the action denoted by the main verb), the Instrument, the Actor's Wearings, some related objects inside the verb event frame, and even those outside. Naturally, the conflation of the Causee with the Actee is the most prevalent one. 


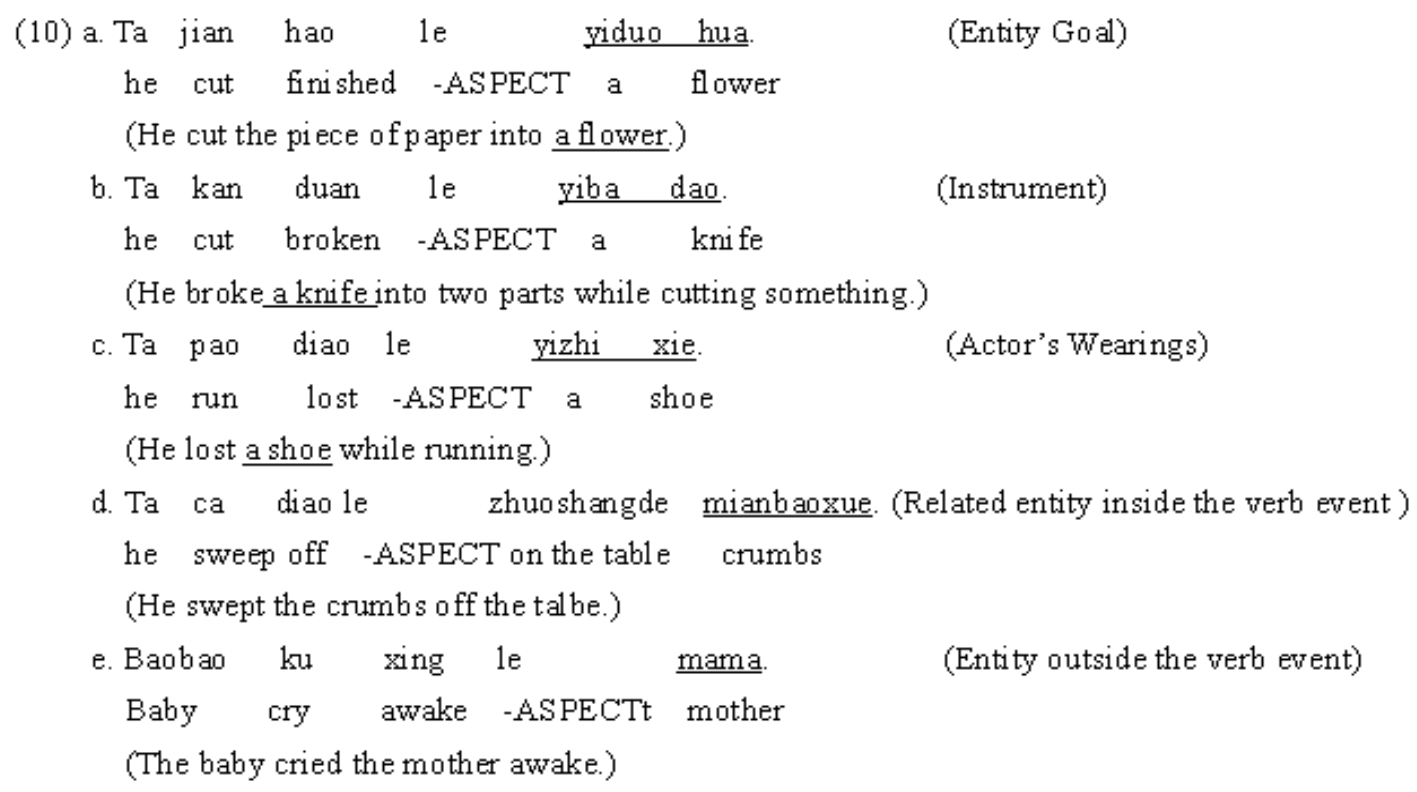

When the Causee is conflated with different participants, it will be construed in different ways and realized in different positions in the grammatical structures, thereby influencing the whole configuration of the verb-resultative constructions.

3.3.1 The Causee Conflated with the Actor as a Whole

When the Causee is conflated with the Actor as whole, there exist two kinds of construal in Chinese verb-resultatives: auto-change through action(as in 11a, 12a) and caused-change through action(as in 11b,12b).

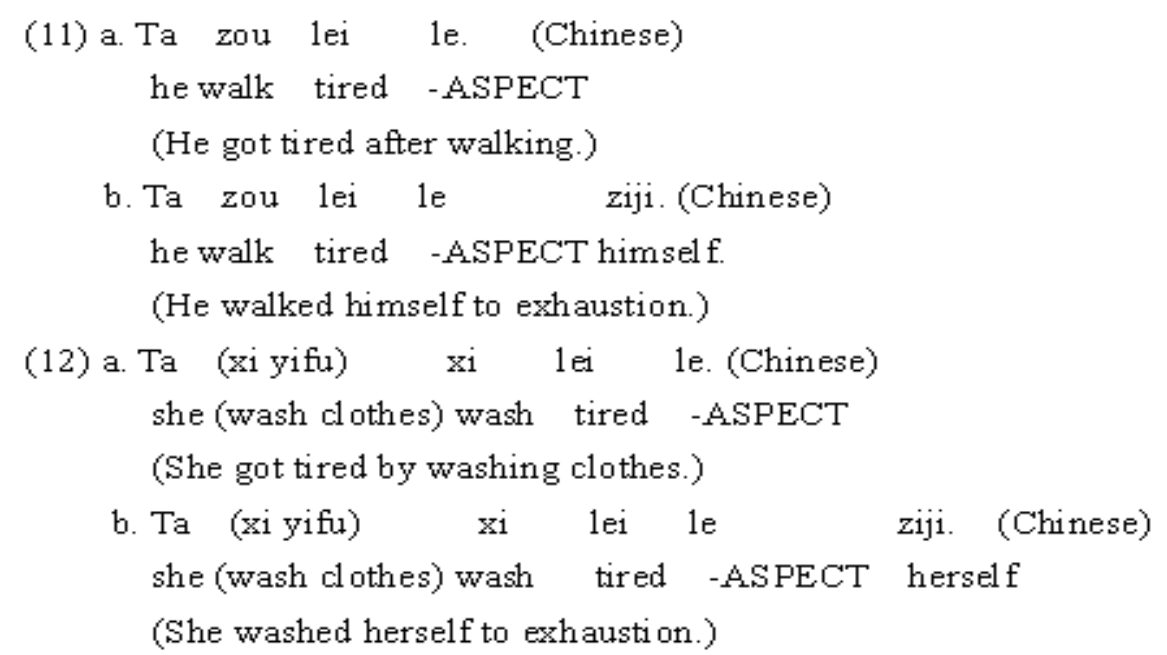

In the auto-change through action, the Actor, which is also the entity undergoing the state change, is realized subject argument, the only argument in the semantic structure, so that the verb-resultative is one-place (11a,12a). In the caused-change through action, the Causer is conflated with the Actor, construed as the Agent in the argument structure, and realized as subject noun phrase, while the Causee is conflated with the Actor as a whole, construed as Patient, and realized as reflective pronoun "ziji" in the object position, so that the verb-resultative is two-place $(11 b, 12 b)$.

However, we notice that there is delicate semantic difference between these two types: the auto-change through action profile the natural change of state, while the caused-change through action profiles that the Actor deliberately overdoes something, leading to a state change of the Actor itself. "The realization of fake reflexives in resultative constructions is a consequence of the fact that under certain circumstances humans perceive their bodies as two separate entities, namely as agents and patients. Since bodies can be construed as patient arguments that are undergoing some change of state instigated by the agent (the conscious Self), the patient has to be explicitly mentioned in order to convey this specific viewpoint." (Boas 2003: 242-43) Only the humans 
with will have such cognitive faculty. When the Actor is inanimate or unwilled, in the condition of unconsciousness, or is actually the Experiencer, it is construed as auto-change through action, not caused-change through action, as in the following sentences:

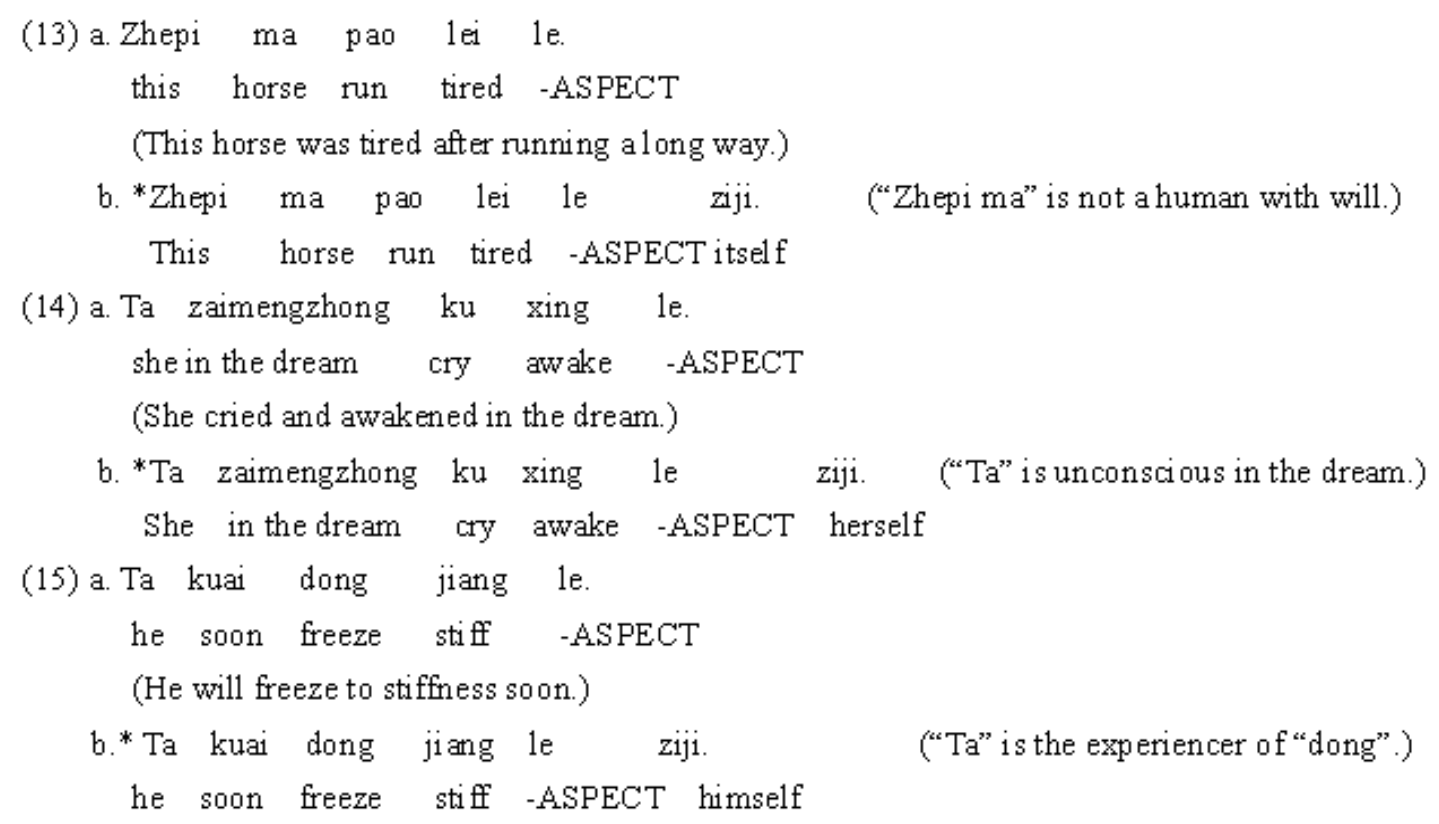

In Chinese verb-resultatives, the Causer can even be conflated with the Actee, construed as agent, realized as subject noun phrase, forming a two-place:

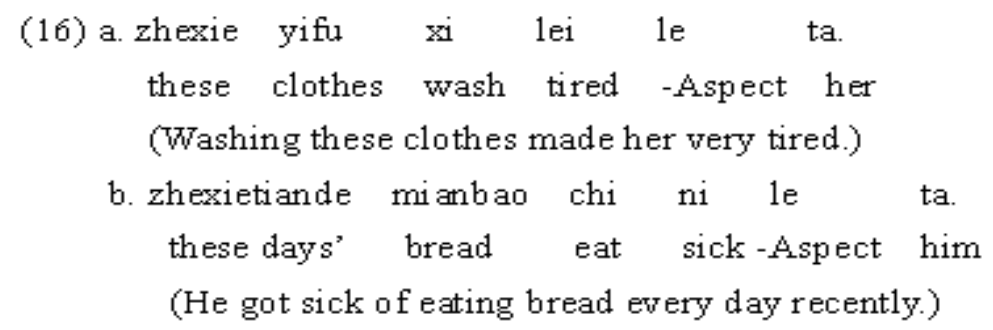

There is another particular type of verb-resultatives. Please consider the following sentences:

$$
\begin{array}{rlllrl}
\text { (17) a. wo kan dong le } & \text { zhexie shu. } \\
\text { I read understand } & \text {-ASPECT these books. }
\end{array}
$$

(I managed to understand these books after reading them.)

b. wo xue hui le zhemen jishu.

I learn grasp -ASPECT this technology

(I have grasped this technology.)

$\begin{array}{lllll}\text { xiao nanhai wan wang le } & \text { yijian shi. } \\ \text { Little boy play forget } & \text {-ASPECT a } & \text { thing }\end{array}$

(The little boy forgot a thing because of being too indulged in playing.)

In sentence (17a), "wo"(I) is not only the Actor of "kan"(read)and "xue"(learn), but also the entity which experiences mental changes ("dong" and "hui"). The noun phrases in the object position ("zhexie shu" and "zhemen jishu") indicate the Actees of "kan" and "xue", not the Causee. Similarly, "xiao nanhai" in (18) is the Actor of "wan" and the entity of "wang", while the object "yijian shi" is not the Actee of the main verb "wan", but the entity which is forgotten. Such verb-resultatives fall into the category of auto-change through action, and they are actually one-place, because the noun phrases in the object position ("zheben shu", "zhemen jishu", and "yijian shi") are a part of the Result-Goal. For example, "dong le zheben shu", "hui le zhemen jishu", and "wang le yijian shi" are the results of "kan", "xue", and "wan" respectively. 


\subsubsection{The Causee conflated with Other Participants}

When the Causee is conflated with other participants (except the Actor as a whole), it is usually realized as patient in the semantic structure, and object noun phrase in the grammatical structure, while the Causer is conflated with the Actor, realized as agent in the semantic structure, and subject in the grammatical structure. And this is the most typical verb-resultative, just as the following:

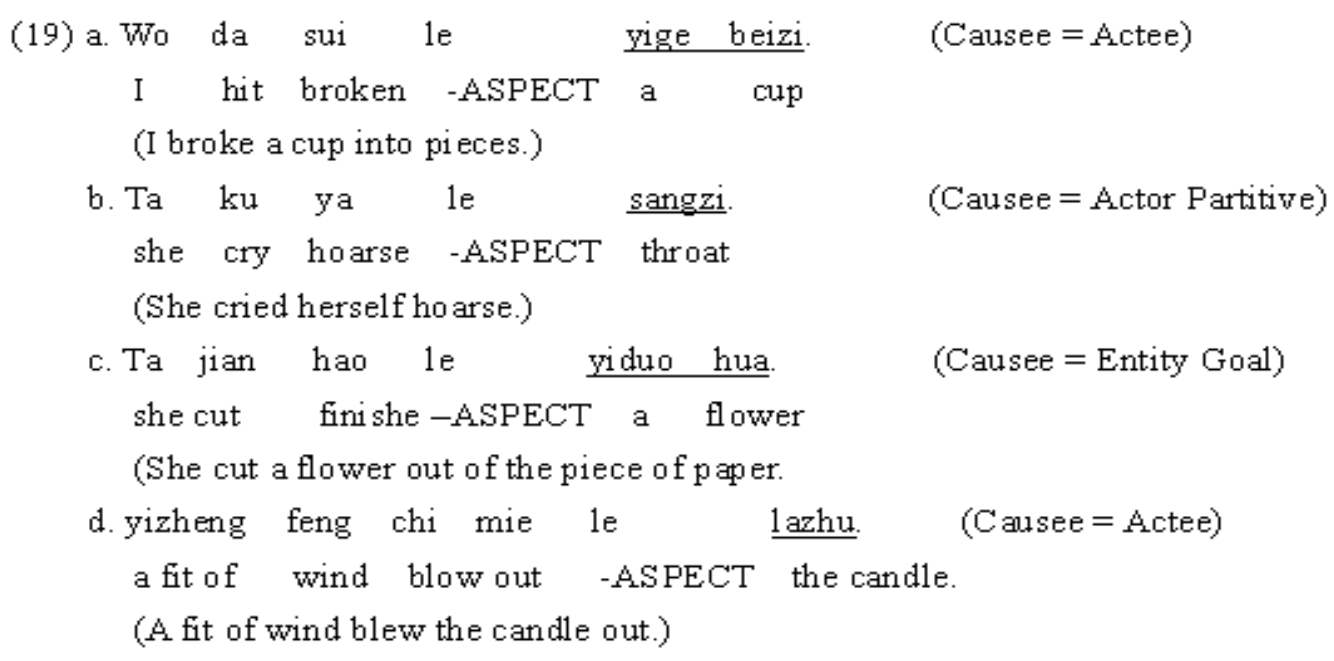

In (19), whichever participant the Causee is conflated with, it is always realized in the object position, and at the same time the subject indicates the conflation of the Causer and the Actor, so the verb-resultative is two-place. The causer can be animate or inanimate (such as "yizheng feng"). However, in some cases, the Causee is realized in the subject position, while the Causer doesn't get realized, such as the following:

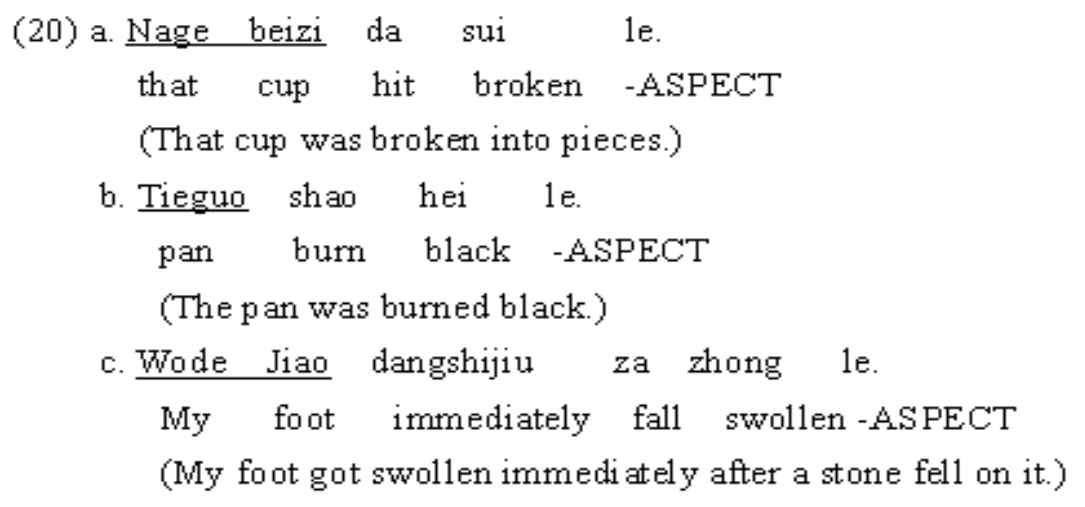

In the verb-resultatives (20) above, the corresponding conceptual structure of action is realized in each sentence, but the conflation of the Causer and the Actor is not grammatically realized. The main reason for this is that the Causee is profiled and realized in the subject position, occupying the space which originally belongs to the semantic role agent. Now, if we want to realize the agent, we must use some marks, such as passive constructions:

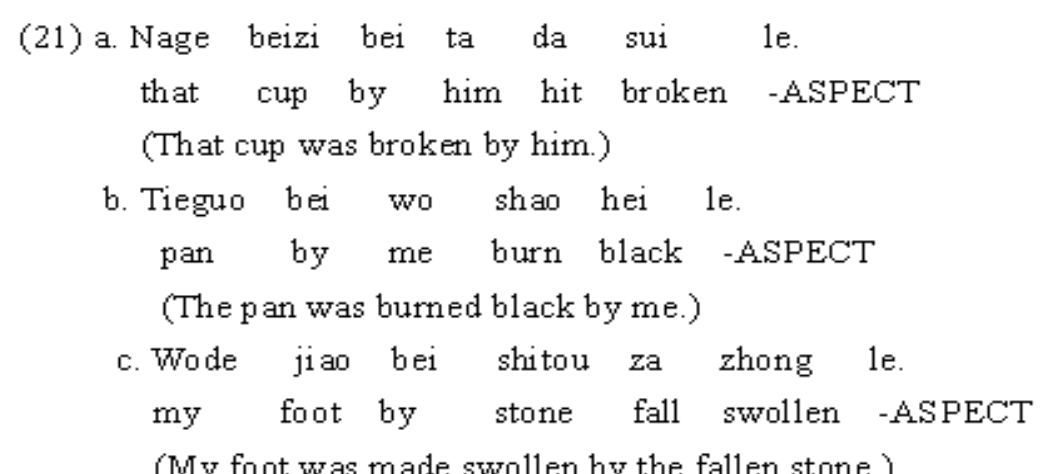

In summary, the semantic structure realization rules of Chinese verb-resultative constructions can be described as 
followed: (i) When the Causee is conflated with the Actor as a whole, there are two construal results: auto-change through action and caused-change through action; the former is realized as one-place(11a, 12a, 17,18), and the latter is realized as two-place. Moreover, the caused-change through action falls into two categories: first, the Causer can be conflated with the Actor, and the Causee is conflated with perceived Actor's self. They are construed respectively as agent and patient in the semantic structure, and realized as subject noun phrase and object reflective(11b, 12b). Second, the Causer can also be conflated with the Actee, while the Causee is conflated with the Actor, and respectively construed as agent and patient in the semantic structure, and subject noun phrase and object noun phrase in the grammatical structure(16). (ii) When the Causee is conflated with other participants except the Actor as a whole, it is always construed as caused-change through action, and realized as two-place (19) or one-place (20). (iii) The entity which undergoes change of state during the action process (i.e. the Causee) must be realized in the semantic structure and the grammatical structure. If it is realized as subject noun phrase, there is no need to realize any other argument in the object position, so that the corresponding verb-resultative is one-place $(11 \mathrm{a}, 12 \mathrm{a}, 17,18,20)$, but the fake reflexive is an exception (11b, $12 \mathrm{~b}$ ). Only when the Causee is realized as object noun phrase is there need to realize other participants (and it must be the Causer) in the subject position, resulting in a two-place verb-resultative (16). (iv) The semantic structures of Chinese verb-resultatives can be divided into three categories: [Agent + Patient + Result-Goal], [Patient + Result-Goal], and [Agent + Result-Goal].

\section{Conclusion}

In the investigation of sentence-level semantic structures, by distinguishing the conceptual content from the grammatical meaning, and representing the sentence-level conceptual content in Conceptual Frames, we can analyze more clearly and accurately the grammatical realization rules of verb-resultative constructions. The conceptual frame of a Chinese verb-resultative construction is typically composed of action conceptual structure and causation conceptual structure; and the participants in these two conceptual structures will be conflated and realized as the relevant argument roles in the semantic structure. When the Causee is conflated with different participants in the action conceptual structure, it can be construed in different ways, and realized in different positions in the corresponding semantic and grammatical structures, thus influencing the whole configuration of the verb-resultative construction.

\section{References}

Boas, H.C. (2003). A constructional approach to resultatives. Stanford, CA: CSLI Publication.

Cheng, Q.L. (2006). Conceptual frame and recognition. Shanghai: Shanghai Foreign Language Education Press.

Chomsky, N. (1981). Lectures on government and binding. Dordrecht: Foris.

Fillmore, C.J. (1971). Types of Lexical Information, In R.D. Steinberg and L.A. Jakobovits (Eds.), Semantics. Cambridge: CUP.

Goldberg, A. E. (1995). Constructions: a construction grammar approach to argument structure. Chicago: The University of Chicago Press.

Jackendoff, R. (1990). Semantic structures. Cambridge, MA: The MIT Press.

Lamb, S. (2004). Language and reality. New York: Continuum International Publishing Group Ltd..

Langacker, R.W. (1998). Conceptualization, symbolization and grammar. In Tomasello. M. (Ed.), The new psychology of language: cognitive and functional approaches to language structure. Mahwah, NJ and London: Erlbaum, pp.1-39.

Levin, B. (1993). English verb classes and alternations. Chicago: University of Chicago Press.

Pinker, S. (1989). Learnability and cognition. Cambridge: MIT Press.

Table 1. The Semantic Frame Activated by the Sentence Fred wiped the table clean.

\begin{tabular}{|c|c|}
\hline sentence & Fred wiped the table clean. \\
\hline Precondition & {$\left[\right.$ Object $_{\text {the table }}+$ State $\left._{\text {clean }}\right]+$ NOT } \\
\hline Conceptual Process & $\begin{array}{l}\text { Actor }_{\text {Fred }}+\text { Action }_{\text {wash }}+\text { Actee }_{\text {the table }} \\
\text { Causer }_{\text {Fred }}+\text { Causation }+ \text { Causee }_{\text {the table }}+\text { Result }_{\text {Object }} \text { the table } \\
\text { clean }]\end{array}$ \\
\hline Postcondition & Object $_{\text {the table }}+$ State $_{\text {clean }}$ \\
\hline
\end{tabular}

